\title{
Introns in Cryptococcus
}

\author{
Guilhem Janbon/+ \\ Institut Pasteur, Unité Biologie des ARN des Pathogènes Fongiques, Département de Mycologie, Paris, France
}

In Cryptococcus neoformans, nearly all genes are interrupted by small introns. In recent years, genome annotation and genetic analysis have illuminated the major roles these introns play in the biology of this pathogenic yeast. Introns are necessary for gene expression and alternative splicing can regulate gene expression in response to environmental cues. In addition, recent studies have revealed that $C$. neoformans introns help to prevent transposon dissemination and protect genome integrity. These characteristics of cryptococcal introns are probably not unique to Cryptococcus, and this yeast likely can be considered as a model for intron-related studies in fungi.

Key words: intron - Cryptococcus - RNA - yeast - alternative splicing

Identified for the first time at the end of the 19th century by Sanfelice in fruit juice (Sanfelice 1894), Cryptococcus is now recognised as a major fungal human pathogen responsible for thousands of deaths every year around the world (Rajasingham et al. 2017). There are two main lineages of pathogenic Cryptococcus, referred to collectively as Cryptococcus complex. Whereas Cryptococcus neoformans affects immunocompromised patients and exists as two varieties (i.e., var. neoformans and var. grubii), Cryptococcus gattii exists in several sub-lineages, some of which can infect immunocompetent patients (Kwon-Chung et al. 2015). Because Cryptococcus lives in the environment (mainly in soil and decaying wood), its biology has been shaped through its interactions with environmental microbiota, as well as seasonal modifications in the physical and chemical soil parameters (May et al. 2016). Much probably, the main Cryptococcus virulence factors, which include capsule and melanin production, have been selected to resist natural environmental predators, such as amoebae and worms (May et al. 2016).

Cryptococcus cells mostly grow as Saccharomyces cerevisiae-like haploid budding yeast, although filamentous or very large multiploid cells can be observed in vitro and in vivo (Zaragoza \& Nielsen 2013). Despite this morphological similarity, Cryptococcus biology and genetics are, in many aspects, different from S. cerevisiae, due in part to the specificity of virulence-associated attributes of $C$. neoformans. For instance, the metabolic pathways and genes required to synthesize polysaccharide capsule and melanin are not present in S. cerevisiae (Moyrand et al. 2007, Alspaugh 2015). Cryptococcus biological specificity also resides in its complex and dynamic transcriptome structure, which in this opportunistic pathogen can be viewed as a method to adapt to different environments.

doi: 10.1590/0074-02760170519

+ Corresponding author: janbon@pasteur.fr

Received 28 November 2017

Accepted 3 January 2018
Introns are probably the most prominent characteristic of the cryptococcal genes. Whereas a typical $S$. cerevisiae gene is intronless, nearly all Cryptococcus genes contain multiple introns (Figure). Recently, these introns and their splicing have been shown to play major roles in gene expression and the control of genetic instability in this pathogenic yeast. Here, I review the current literature surrounding intron structural characteristics and evolutionary conservation, as well as the roles of introns in the Cryptococcus biology.

Intron position and structure - Historically, the early cloning and sequencing of Cryptococcus genes revealed the presence of multiple introns. In 1990, Edman and Kwon-Chung cloned the gene encoding $C$. neoformans orotidine monophosphate pyrophosphorylase (URA5) and identified two small introns of 52 and 49 nucleotides (Edman \& Kwon-Chung 1990). The gene encoding dihydrofolate reductase was cloned three years later by JC Edman and displayed a similar structure with two short introns (Sirawaraporn et al. 1993). All Cryptococcus genes subsequently cloned contain at least one intron, suggesting that all genes in this pathogenic yeast contain at least one intron. Of note, the phosphoribosylanthranilate isomerase-encoding gene (TRPI) was originally reported to be intronless (Perfect et al. 1992) but does indeed contain two short introns within its sequence (http://fungidb.org/ fungidb/app/record/gene/CNAG_04501) (Stajich et al. 2012). Large-scale sequencing of cDNA molecules and the more recent use of RNA-Seq data confirmed the complex structure of cryptococcal genes (Kupfer et al. 2004, Loftus et al. 2005, Janbon et al. 2014, González-Hilarion et al. 2016, Ferrareze et al. 2017). Each gene contains an average of 5.7 introns. These introns are mostly present in coding sequence (CDS), although some are present in the 5' and 3' UTR regions. This is in sharp contrast to $S$. cerevisiae, in which only 283 genes contain an intron (Juneau et al. 2007, Janbon et al. 2014). In C. neoformans var. grubii, introns also have been identified in transcribed active regions with no obvious coding potential (miscRNAs) (Janbon et al. 2014). Overall, the C. neoformans genomes contain more than 40,000 introns. With 3.35 introns per $\mathrm{kb}$ of coding sequence, C. neoformans is the most intron- 


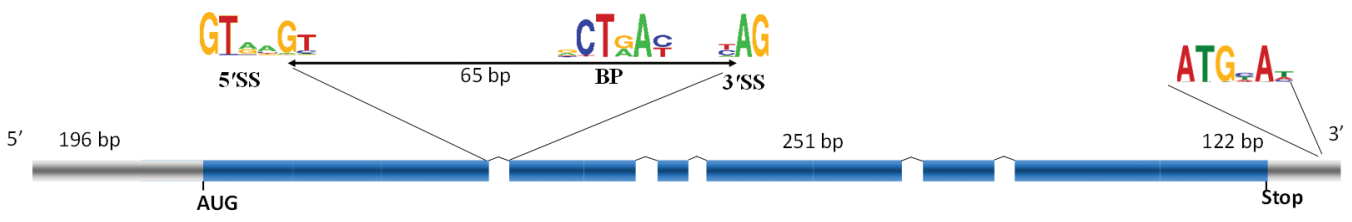

Schematic representation of a typical Cryptococcus gene. The coding sequence (CDS) is represented in blue whereas the untranscribed regions (UTR) are in grey. The consensus sequences associated with the 5' splice site (5'SS) 3' splice site (3'SS) and branching point (BP) are indicated. The average size of the introns, exons, 3' and 5'UTR regions are given.

rich fungal species for which a reliable genome annotation is available (Stajich et al. 2007, Janbon et al. 2014). It is important to note here that the presence of introns in fungal genes is probably more the rule than the exception. In that sense, $C$. neoformans can be considered to be a model for intron-related studies in fungi (Stajich et al. 2007).

As in all fungi, Cryptococcus introns are relatively small, with an average size of $65 \mathrm{nt}$; the UTR introns are slightly larger than those in the CDS. The analysis of $C$. neoformans and $C$. gattii intron length suggests strong but complex evolutionary selection of intron size (Hughes et al. 2008, Janbon et al. 2014); thus, the size of UTR introns, as well as the first and last introns within the CDS, seem to be constrained by different selective pressures, suggesting potential specific roles or splicing patterns (Hughes et al. 2008). As shown in Figure, Cryptococcus introns are associated with specific consensus motifs with very minor differences between species (Kupfer et al. 2004, Irimia et al. 2009, Janbon et al. 2014). However, in contrast to S. cerevisiae, in which splicing signals conform closely to consensus, signals in $C$. neoformans are more degenerated, resembling metazoan introns in this respect. Interestingly, a metazoan-like bias in amino acid and codon usage at the C. neoformans 3' and 5' exon ends has been also observed (Warnecke et al. 2008). Again, this bias was not observed in S. cerevisiae or Schizosaccharomyces pombe, suggesting specific exon-based splicing regulation in $C$. neoformans. Consistent with this model, $C$. neoformans proteome contains multiple homologues of SR or SR-like proteins known to be involved in splicing an alternative splicing regulation in metazoans but not typically found in single cell eukaryotes [(Warnecke et al. 2008, Dumesic et al. 2013); Janbon, unpublished observations]. Although mechanistic analyses are yet to be performed, it is tempting to put these structural observations in perspective with the abundance and complex regulation of alternative splicing recently reported in $C$. neoformans (González-Hilarion et al. 2016).

Intron gain and loss - The evolutionary dynamics of introns have been widely studied in fungi, including $C$. neoformans (Nielsen et al. 2004, Sharpton et al. 2008, Croll \& McDonald 2012). These studies, all based on genome sequence analyses, identified few examples of intron gain and loss between the Cryptococcus lineages (Roy 2009, Croll \& McDonald 2012). Stajich and Dietrich identified a $C$. neoformans var. grubii gene from which 10 adjacent introns have been lost, suggesting an mRNA-based mechanism (Stajich \& Dietrich 2006). Conversely, in 2009 Row found evidence for five cases of intron gain in the $C$. neoformans var. neoformans genome (Roy 2009). These are recent events, as $C$. neoformans var. grubii and $C$. neoformans var. neoformans diverged only 37 million years ago (Kwon-Chung et al. 2015). The recent genome re-annotation of the two $C$. neoformans varieties based on RNA-Seq data gave us the opportunity to gain insight into intron dynamics. We identified 6,241 orthologous gene couples and analysed intron conservation between the varieties (GonzálezHilarion et al. 2016). Whereas half of the introns in the 5'UTR and $90 \%$ in the 3'UTR were either lost or at a different position, the overwhelming majority $(99.5 \%)$ of introns in the CDS were present in the same position in both $C$. neoformans varieties, confirming results of previous studies (Roy 2009, Croll \& McDonald 2012, González-Hilarion et al. 2016). Overall, these data revealed that intron gain and loss are very rare events, suggesting a strong selective pressure to maintain intron richness in Cryptococcus genes.

Biological importance - Introns play a central role in the regulation of gene expression in C. neoformans, which may explain the strong selective pressure in favor of introns in this species. Indeed, in situ replacement of a wild-type multi-exonic gene by an intronless version of the same gene is usually associated with a strong decrease in gene expression (Dumesic et al. 2013, Goebels et al. 2013). In some cases, intron deletion completely abolishes gene expression (Goebels et al. 2013). Our analysis on a model gene suggests that most introns play a cumulative positive role probably because their splicing promotes mRNA export out of the nucleus (Goebels et al. 2013); in the absence of introns, mRNA molecules would be poorly exported and instead degraded by nuclear exonucleases. It is interesting to note that only 35 expressed genes in $C$. neoformans var. grubii are intronless, further supporting the idea that introns play a central role in the regulation of gene expression (Janbon et al. 2014). Furthermore, indirect evidence suggests that incompletely spliced mRNA molecules remain in the nucleus, where they are degraded (González-Hilarion et al. 2016). Accordingly, poorly spliced introns play a negative role in the regulation of gene expression (Goebels et al. 2013), and incompletely spliced mRNAs containing a premature termination codon are not degraded by the nonsense-mediated mRNA decay cytoplasmic pathway (González-Hilarion et al. 2016). Interestingly, genome-wide and sin- 
gle-gene studies revealed that alternative splicing (mostly intron retention) affects most Cryptococcus genes (Loftus et al. 2005, Missall et al. 2005, González-Hilarion et al. 2016). In addition, RNA-Seq analyses strongly suggest that alternative splicing is tightly and specifically regulated by environmental cues (González-Hilarion et al. 2016). Thus, in C. neoformans and probably in most fungi, alternative splicing is certainly not a major source of proteome diversification, but it should be considered an additional layer of gene expression regulation providing a mechanism by which each cell finely tunes gene expression levels in response to environmental modifications (González-Hilarion et al. 2016).

Introns and intron splicing prevent genomic instability in C. neoformans. For example, intron splicing efficiency regulates the expression of transposon-related genes in C. neoformans. Dumesic and colleagues demonstrated that inefficiently spliced mRNAs, which are typically transcribed based on transposable sequences, are targeted by the Spliceosome-Coupled and Nuclear RNAi complex (SCANR), becoming a template for dsRNA synthesis and siRNA production (Dumesic et al. 2013). Accordingly, this RNAi-dependent nuclear mechanism also involves the lariat debranching enzyme Dbrl. Dumesic and colleagues also reported that transposonrelated transcripts are more likely to be associated with the spliceosome, suggesting that their introns are poorly spliced. Thus, C. neoformans introns and their splicing limit the expression of transposon-related genes and prevent transposon dissemination in the genome.

Another emerging mechanism by which introns may protect genomes is by preventing DNA-RNA hybrid (R-loop) formation in the wake of the RNA polymerase II, thus avoiding the associated accumulation of DNA damage (Bonnet et al. 2017). Bonnet and colleagues analysed phenotypes associated with deletion of the conserved THO complex component encoding the gene HPR1 which is known to prevent R-loop formation in $S$. cerevisiae and human cells (Domínguez-Sánchez et al. 2011). They found that this mutation has very little impact on growth and R-loop formation in intron-rich organisms such as C. neoformans, but it is associated with a strong growth defect and marked increase of R-loop formation in intron-poor yeasts such as Candida glabrata and $S$. cerevisiae. They also showed that introns and spliceosome-dependent mRNP assembly, but not splicing per se, prevent R-loop formation and genome instability (Bonnet et al. 2017). Altogether, these studies reveal the central role of introns in Cryptococcus species for control of genome stability.

Summary and future directions - In summary, these studies have revealed introns as key features in the biology of Cryptococcus and, in the maintenance of their genome integrity. They also suggest that their structure have been finely selected throughout evolution. Nevertheless, a number of questions remain to be answered. (1) For instance, the mechanisms regulating intron gain and loss in fungi is still a very active domain of research. A better description of intron structure and position in the different lineages of C. gattii and C. neoformans (Kwon-
Chung et al. 2015) will likely provide insights into this matter. Similarly, although many genomes have been sequenced in recent years (Desjardins et al. 2017), a precise comparison of intron position within each lineage would identify recent intron gain or loss events. In addition, recent reports identified independent links between the biology of introns and genomic stability in Cryptococcus, thus paving new avenues for genome structure evolution in fungi. (2) Additionally, the role of introns in gene regulation requires further analyses. The current model on Cryptococcus intron retention-dependent regulation of gene expression (González-Hilarion et al. 2016) suggests the existence of complex networks of transduction pathways and RNA binding proteins that precisely regulate alternative splicing in response to environmental cues. However, no elements within these networks have been identified, and deciphering the mechanisms regulating alternative splicing will represent a major challenge in future studies. (3) Furthermore, intron biology is intertwined with $C$. neoformans RNA biology. For example, as previously reported in metazoans several alternative polyadenylation sites within introns have been identified (Janbon et al. 2014). In metazoans, polyadenylation and splicing are there in competition and their alternative usage results in transcripts of different size, stability and sometime coding potential (Tian \& Manley 2017). In fungi and more specifically in $C$. neoformans the biological consequences of this choice remain to be studied. The roles of introns in the UTRs of number of Cryptococcus genes are mainly unknown. In metazoans, introns in UTRs were ignored for a long time but they have recently been shown to play important and unique roles in the regulation of gene expression (Bicknell et al. 2012). In Cryptococcus, the specificity of their structure and evolutionary conservation suggest also unique functions which remains to be identified. (4) Finally, introns have been identified in miscRNAs, but the function, splicing, and evolutionary conservation of this intron subclass have not been studied yet.

\section{ACKNOWLEDGEMENTS}

To Cecelia Shertz Wall for editing the manuscript.

\section{REFERENCES}

Alspaugh JA. Virulence mechanisms and Cryptococcus neoformans pathogenesis. Fungal Genet Biol. 2015; 78: 55-8.

Bicknell AA, Cenik C, Chua HN, Roth FP, Moore MJ. Introns in UTRs: why we should stop ignoring them. Bioessays. 2012; 34(12): 1025-34.

Bonnet A, Grosso AR, Elkaoutari A, Coleno E, Presle A, Sridhara $\mathrm{SC}$, et al. Introns protect eukaryotic genomes from transcriptionassociated genetic instability. Mol Cell. 2017; 67(4): 608-21.

Croll D, McDonald BA. Intron gains and losses in the evolution of $F u$ sarium and Cryptococcus fungi. Genome Biol Evol. 2012; 4(11): 1148-61.

Desjardins CA, Giamberardino C, Sykes SM, Yu C-H, Tenor JL, Chen Y, et al. Population genomics and the evolution of virulence in the fungal pathogen Cryptococcus neoformans. Genome Res. 2017; 27(7): 1207-19.

Domínguez-Sánchez MS, Barroso S, Gómez-González B, Luna R, Aguilera A. Genome instability and transcription elongation im- 
pairment in human cells depleted of THO/TREX. PLoS Genet. 2011; 7(12): e1002386

Dumesic PA, Natarajan P, Chen C, Drinnenberg IA, Schiller BJ, Thompson JD, et al. Stalled spliceosomes are a signal for RNAimediated genome defense. Cell. 2013; 152(5): 957-68.

Edman JC, Kwon-Chung KJ. Isolation of the URA5 gene from Cryptococcus neoformans var. neoformans and its use as a selective marker for transformation. Mol Cell Biol. 1990; 10(9): 4538-44.

Ferrareze PAG, Streit RSA, dos Santos PR, dos Santos FM, de Almeida RMC, Schrank A, et al. Transcriptional analysis allows genome reannotation and reveals that Cryptococcus gattii VGII undergoes nutrient restriction during infection. Microorganisms. 2017; 5(3): E49.

Goebels C, Thonn A, González-Hilarion S, Rolland O, Moyrand F, Beilharz $\mathrm{TH}$, et al. Introns regulate gene expression in Cryptococcus neoformans in a Pab2p dependent pathway. PLoS Genet. 2013; 9(8): e1003686.

González-Hilarion S, Paulet D, Lee K-T, Hon C-C, Lechat P, Mogensen $\mathrm{E}$, et al. Intron retention-dependent gene regulation in Cryptococcus neoformans. Sci Rep. 2016; 6: 32252.

Hughes SS, Buckley CO, Neafsey DE. Complex selection on intron size in Cryptococcus neoformans. Mol Biol Evol. 2008; 25(2): 247-53.

Irimia M, Roy SW, Neafsey DE, Abril JF, Garcia-Fernández J, Koonin EV. Complex selection on 5' splice sites in intron-rich organisms. Genome Res. 2009; 19(11): 2021-7.

Janbon G, Ormerod KL, Paulet D, Byrnes III EJ, Chatterjee G, Yadav $\mathrm{V}$, et al. Analysis of the genome and transcriptome of Cryptococcus neoformans var. grubii reveals complex RNA expression and microevolution leading to virulence attenuation. PLoS Genet. 2014; 10(4): e1004261.

Juneau K, Palm C, Miranda M, Davis RW. High-density yeast-tiling array reveals previously undiscovered introns and extensive regulation of meiotic splicing. Proc Natl Acad Sci USA. 2007; 104(5): 1522-7.

Kupfer DM, Drabenstot SD, Buchanan KL, Lai H, Zhu H, Dyer DW, et al. Introns and splicing elements of five diverse fungi. Eukaryot Cell. 2004; 3(5): 1088-100.

Kwon-Chung KJ, Fraser JA, Doering TL, Wang Z, Janbon G, Idnurm A, et al. Cryptococcus neoformans and Cryptococcus gattii, the etiologic agents of cryptococcosis. Cold Spring Harb Perspect Med. 2015; 4(7): a019760.

Loftus B, Fung E, Roncaglia P, Rowley D, Amedeo P, Bruno D, et al. The genome and transcriptome of Cryptococcus neoformans, a basidiomycetous fungal pathogen of humans. Science. 2005; 307(5713): 1321-4.
May RC, Stone NRH, Wiesner DL, Bicanic T, Nielsen K. Cryptococcus: from environmental saprophyte to global pathogen. Nat Rev Microbiol. 2016; 14(2): 106-17.

Missall TA, Moran JM, Corbett JA, Lodge JK. Distinct stress responses of two functional laccases in Cryptococcus neoformans are revealed in the absence of the thiol-specific antioxidant Tsal. Eukaryot Cell. 2005; 4(1): 202-8.

Moyrand F, Fontaine T, Janbon G. Systematic capsule gene disruption reveals the central role of galactose metabolism on Cryptococcus neoformans virulence. Mol Microbiol. 2007; 64(3): 771-81.

Nielsen CB, Friedman B, Birren B, Burge CB, Galagan JE. Patterns of intron gain and loss in fungi. PLoS Biol. 2004; 2(12): e422.

Perfect JR, Rude TH, Penning LM, Johnson SA. Cloning the Cryptococcus neoformans TRP1 gene by complementation in Saccharomyces cerevisiae. Gene. 1992; 122(1): 213-7.

Rajasingham R, Smith RM, Park BJ, Jarvis JN, Govender NP, Chiller TM, et al. Global burden of disease of HIV-associated cryptococcal meningitis: an updated analysis. Lancet Infect Dis. 2017; 17(8): 873-81.

Roy SW. Intronization, de-intronization and intron sliding are rare in Cryptococcus. BMC Evol Biol. 2009; 9: 192-2.

Sanfelice F. Contributo alla morphologia e biologica dei blastomiceti che sisciluppano nei succhi di alcuni frutti. Ann Ist Ig R Univ Roma. 1894; 4: 463-9.

Sharpton TJ, Neafsey DE, Galagan JE, Taylor JW. Mechanisms of intron gain and loss in Cryptococcus. Genome Biol. 2008; 9(1): R24.

Sirawaraporn W, Cao M, Santi DV, Edman JC. Cloning, expression, and characterization of Cryptococcus neoformans dihydrofolate reductase. J Biol Chem. 1993; 268(12): 8888-92.

Stajich JE, Dietrich FS, Roy SW. Comparative genomic analysis of fungal genomes reveals intron-rich ancestors. Genome Biol. 2007; 8(10): R223.

Stajich JE, Dietrich FS. Evidence of mRNA-mediated intron loss in the human-pathogenic fungus Cryptococcus neoformans. Eukaryot Cell. 2006; 5(5): 789-93.

Stajich JE, Harris T, Brunk BP, Brestelli J, Fischer S, Harb OS, et al. FungiDB: an integrated functional genomics database for fungi. Nucleic Acids Res. 2012; 40(Database issue): D675-81.

Tian B, Manley JL. Alternative polyadenylation of mRNA precursors. Nat Rev Mol Cell Biol. 2017; 18(1): 18-30.

Warnecke T, Parmley JL, Hurst LD. Finding exonic islands in a sea of non-coding sequence: splicing related constraints on protein composition and evolution are common in intron-rich genomes. Genome Biol. 2008; 9(2): R29.

Zaragoza O, Nielsen K. Titan cells in Cryptococcus neoformans: cells with a giant impact. Curr Opin Microbiol. 2013; 16(4): 409-13. 